\title{
MicroRNA-7a2 suppression causes hypogonadotropism and uncovers signaling pathways in gonadotropes
}

\author{
William F. Crowley and Ravi Balasubramanian \\ Harvard Reproductive Endocrine Sciences Center, Department of Medicine, Massachusetts General Hospital, and Harvard Medical School, Boston, Massachusetts, USA.
}

\begin{abstract}
MicroRNAs (miRNAs) have emerged as important regulators of a variety of biological processes and pathways. In this issue of the $J C I$, Ahmed et al. reveal that miR-7a2 is a critical regulator of sexual maturation and reproductive function, as mice lacking miR-7a2 develop hypogonadotropic hypogonadism and infertility. Using a bioinformatics approach, the authors identified several miR-7a2 target genes and pathways that have not been previously associated with gonadotropin biosynthesis and/or secretion. Together, these results identify miR-7a2-regulated genes involved in reproductive hormone biosynthesis pathways and provide a framework for future studies aimed at understanding rare reproductive conditions.
\end{abstract}

The microRNA-7 family

MicroRNAs (miRNAs) have diverse roles in a number of biological processes; however, many functions of these regulators remain largely a mystery. In this issue, Ahmed et al. provide new functional information about the miR-7 family, a small but conserved family of noncoding RNAs that consists of three members - miR-7a1, miR-7a2, and miR-7b (1). Members of the miR-7 family are expressed throughout neuronal development and exhibit a particularly robust expression in select neuroendocrine cells, most notably in cells of the mouse anterior pituitary. Then, at various stages of development, these miRNAs target a series of miRNA-response elements that begin to be expressed primarily in the $3^{\prime}$ UTRs of several neuroendocrine genes that are key for subsequent anterior pituitary development. Collectively, this chain of developmental events sets the stage for these miRNAs to bind developmental genes and reverse the repression of several "downstream" genes and allow precise control of protein expression that they have been sustaining. These dynamic changes carefully orchestrate the delicate pas de deux that constitutes organ differentiation.
Using clever genetic and biochemical approaches to perturb the miR-7 family in the murine hypothalamus and pituitary across developmental stages and during adulthood, Ahmed et al. have established that, among these three miR-7 precursors, only miR-7a2 is highly enriched in the pituitary. Additionally, only loss of miR-7a2 function produced selective hypogonadotropism as the result of suppression of the biosynthesis and secretion of follicle-stimulating hormone (FSH) and the genes encoding the $\alpha$ and $\beta$ subunits of luteinizing hormone $(\mathrm{LH})$ in mouse gonadotropes. The ensuing gonadotropin deficiency produced profound downstream hypogonadism in both sexes that was reversed by exogenous gonadotropin administration in female miR-7a2-deficient mice, thus eliminating the possibility of any intrinsic gonadal component to this hypogonadism. In addition to the specificity of this hypogonadotropic hypogonadism to the loss of miR-7a2 and not to other members of the miRNA-7 family, the hypogonadotropism was secondary to defects selective for the anterior pituitary, with no evidence of hypothalamic gonadotropin-releasing hormone $(\mathrm{GnRH})$ defects.

Related Article: p. 1061

Conflict of interest: The authors have declared that no conflict of interest exists

Reference information: J Clin Invest. 2017;127(3):796-797. https://doi.org/10.1172/JCI92846

miR-7a2 targets and gonadotropin biosynthesis pathways

Further, Ahmed et al. identified potential miR-7a2 targets responsible for the miR$7 \mathrm{a} 2$ deficiency-related hypogonadotropism by applying RNA sequencing to transcripts from the pituitary of miR-7a2-deficient animals compared with controls. Bioinformatic analysis revealed tissue-specific upregulation of two genes: Ptgfrn, which encodes a negative regulator of prostaglandin receptor F2a, and golgi glycoprotein 1 (Glg1), a member of the cysteine-rich FGF receptor family. In addition, miR-7a2 KO mice displayed a drastic reduction in gremlin 1 (Grem1), a secreted bone morphogenetic protein 4 (BMP4) antagonist whose reduction was mediated in part by disinhibition of GLG1, consequently leading to increased BMP activity, which is known to affect pituitary development and impair gonadotrope function $(2,3)$. Furthermore, among the 55 most regulated miR-7 targets, silencing of Ptgfrn and Glg1 in gonadotrope-specific pituitary cell lines induced hormone expression, indicating that these genes act as negative regulators of pituitary hormone transcription. Taken together, these results uncover at least two previously unappreciated signaling pathways that appear to be obligate intermediates, at least in this clever new model of selective hypogonadotropism. Neither the TGF- $\beta$ /BMP4 axis nor the prostaglandin-signaling pathway has drawn much attention as a major player in gonadotropin biosynthesis and/or secretion. The findings of Ahmed et al. now provide important new leads on potential intermediates in gonadotropin biosynthesis pathways that will require considerable follow-up. However, the study by Ahmed and colleagues represents novel findings that seem to be internally coherent and robust across multiple biological approaches.

In addition to providing insights into gonadotropin biosynthesis pathways, the current study by Ahmed et al. provides an important contribution to the growing 
genetic evidence for the role of miRNAs in mammalian reproduction. LIN28B, which encodes an RNA-binding protein that inhibits the maturation of let7 miRNAs, has previously been implicated in the timing of menarche in humans (4). miRNA-200/429 and miRNA-155 were recently shown to be a part of a complex developmental switch that controls Gnrh promoter activity that is essential for the normal initiation of murine puberty (5). Gonadotrope-specific deletion of dicer in mice results in severely suppressed gonadotropins and fertility defects (6). And finally, female mice lacking miR-200b and miR-429 have been shown to exhibit hypogonadotropism and anovulation (7).

\section{Conclusions}

In attempting to translate these murine findings to human disease, it is important to take the following into consideration. Patients suffering from idiopathic hypogonadotropic hypogonadism (IHH) generally exhibit a defect in either the secretion of $\mathrm{GnRH}$ from the hypothalamus and/or its action at the level of the anterior pituitary, such as has been observed in a human and murine model of GnRH deficiency (8). However, this rare clinical entity can also be associated with anosmia, a defect that occurs in approximately $50 \%$ to $60 \%$ of patients and generally signals a developmental failure in the migration or differentiation of the GnRH neurons in their travels from their embryonic origins in the olfactory placode to their final anatomic destination within the hypothalamus. In contrast, normosmic IHH patients have a normal olfactory system with normal sense of smell, and it is into this group that the small subset of patients with a pituitary defect fit. While the frequency of normosmic IHH may be rare, the novelty of this phenotype, like all new gene discoveries in the reproductive pathways of humans, is high. In this regard, Ahmed et al. propose that miR-7a2 and its downstream target genes are putative candidates for causing this rare subset of IHH patients with pituitary defects. While speculative, when successful, such candidate gene approaches can illuminate entirely new domains of reproductive biology that beg to be explored. The recent discovery of the critical involvement of the ubiquitination pathway as a cause of the Gordon Holmes variant of hypogonadotropic hypogonadism in humans is an example of this phenomenon, where applying genetic approaches to a rare but "prismatic" subset of $\mathrm{IHH}$ patients with pituitary defects has provided unique insights into previously unappreciated and critical roles for the ubiquitination pathway in gonadotrope function (9). In this regard, the advantage of human genetics is considerable, as candidates that emerge from these basic experiments can now be queried in genomic sequencing data, which are currently being generated by several groups from humans with various forms of $\mathrm{IHH}$.

In addition to contributing new knowledge and leads for other basic scientists to follow, studies such as that by Ahmed et al. provide potential targets for mapping to human disorders by clinical investigators. In addition, these studies offer potential diagnostic and therapeutic opportunities for our biotechnology and pharmaceutical colleagues seeking to either develop treatments for subsets of infertile patients (so-called personalized medicine) or novel approaches to contraception.

\section{Acknowledgments}

WFC is supported by National Institute of Child Health and Human Development
(NICHD) funding (P50: HD028138) of a P50 Center of Excellence in Translational Research in Fertility and Infertility. RB is supported by a NICHD K23 Career Development Award (K23: HD077043)

Address correspondence to: William F. Crowley, Bartlett Hall Extension 511, Massachusetts General Hospital, Boston, Massachusetts 02114, USA. Phone: 617.726.5390; E-mail: crowley.william@ mgh.harvard.edu.

1. Ahmed K, et al. Loss of microRNA-7a2 induces hypogonadotropic hypogonadism and infertility. JClin Invest. 2017;127(3):1061-1074.

2. Nicol L, Faure MO, McNeilly JR, Fontaine J, Taragnat C, McNeilly AS. Bonemorphogenetic protein- 4 interacts with activin and GnRH to modulategonadotrophin secretion in L $\beta$ T2 gonadotrophs. J Endocrinol. 2008;196(3):497-507.

3. Massagué J, Blain SW, Lo RS. TGF $\beta$ signaling in growth control, cancer, and heritable disorders. Cell. 2000;103(2):295-309.

4. Ong KK, et al. Genetic variation in LIN28B is associated with the timing of puberty. Nat Genet. 2009;41(6):729-733.

5. Messina A, et al. A microRNA switch regulates the rise in hypothalamic GnRH production before puberty. Nat Neurosci. 2016;19(6):835-844.

6. Wang $\mathrm{H}$, et al. Gonadotrope-specific deletion of Dicer results in severely suppressed gonadotropins and fertility defects. J Biol Chem. 2015;290(5):2699-2714.

7. Hasuwa H, Ueda J, Ikawa M, Okabe M. miR$200 \mathrm{~b}$ and $\mathrm{miR}-429$ function in mouse ovulation and are essential for female fertility. Science. 2013;341(6141):71-73.

8. Balasubramanian R, Crowley WF Jr. Isolated GnRH deficiency: a disease model serving as a unique prism into the systems biology of the GnRH neuronal network. Mol Cell Endocrinol. 2011;346(1-2):4-12.

9. Margolin DH, et al. Ataxia, dementia, and hypogonadotropism caused by disordered ubiquitination. $N$ Engl J Med. 2013;368(21):1992-2003. 